\section{Fatores associados ao desenvolvimento infantil em crianças brasileiras: linha de base da avaliação do impacto do Programa Criança Feliz}

\section{Factors associated infant development in Brazilian children: baseline of the impact assessment of the Happy Child Program}

\author{
Factores asociados al desarrollo infantil en niños \\ brasileños: base de referencia para la evaluación \\ del impacto del Programa Niño Feliz
}

\author{
Tiago N. Munhoz 1,2 \\ Iná S. Santos 2 \\ Cauane Blumenberg 2 \\ Raquel Siqueira Barcelos 2 \\ Caroline C. Bortolotto 2 \\ Alicia Matijasevich 3 \\ Hernane G. Santos Júnior 4 \\ Letícia Marques dos Santos 5 \\ Luciano L. Correa 6 \\ Marta Rovery de Souza 7 \\ Pedro I. C. Lira 8 \\ Elisa Rachel Pisani Altafim 9 \\ Esmeralda Correa Macana 10 \\ Cesar G. Victora 2
}

\section{Resumo}

O objetivo deste artigo foi avaliar os fatores socioeconômicos, familiares e individuais associados ao desenvolvimento infantil no primeiro ano de vida, entre famílias em vulnerabilidade social. Trata-se de uma análise transversal, com dados da linha de base de um ensaio randomizado. O estudo incluiu 3.242 crianças < 12 meses de idade, residentes em 30 municípios de cinco regiões do Brasil. A escolha de estados e municípios foi intencional, tendo como base a implementação do Programa Criança Feliz. A amostra foi selecionada a partir de crianças elegíveis para o Programa Criança Feliz, cujo objetivo é promover a estimulação e o desenvolvimento infantil. O Ages and Stages Questionnaire (ASQ) foi utilizado para avaliação do desenvolvimento infantil. Um modelo de análise multinível em três níveis (estado, município e indivíduos), usando teste de Wald para heterogeneidade e tendência linear, estimou a média do ASQ-3 e intervalo de 95\% de confiança (IC95\%). Análises foram ajustadas para potenciais confundidores. Foram analisadas informações de 3.061 (94,4\%) crianças com dados disponíveis para ASQ-3. Escores de desenvolvimento infantil (total e em todos os dominios) foram cerca de $12 \%$ menores em crianças nascidas pré-termo e com restrição do crescimento intrauterino (pequenas para idade gestacional). Observou-se menores escores em filhos de mães com baixa escolaridade, com sintomas de depressão, com duas ou mais crianças menores de sete anos residindo no domicílio e que não relataram autopercepção de apoio/ajuda durante a gestação. Conclui-se que características potencialmente modificáveis (escolaridade, depressão materna e prematuridade/restrição do crescimento intrauterino) apresentaram maior impacto na redução do escore de desenvolvimento em todos os domínios avaliados.
Correspondência

T. N. Munhoz

Curso de Psicologia, Universidade Federal de Pelotas. Rua Marechal Deodoro 1160, Pelotas, RS 96020-220, Brasil. tiago.munhoz@ufpel.edu.br

${ }_{1}$ Curso de Psicologia, Universidade Federal de Pelotas, Pelotas, Brasil.

2 Programa de Pós-graduação em Epidemiologia, Universidade Federal de Pelotas, Pelotas, Brasil.

3 Faculdade de Medicina, Universidade de São Paulo, São Paulo, Brasil.

${ }_{4}$ Instituto de Saúde Coletiva, Universidade Federal do Oeste do Pará, Santarém, Brasil.

5 Instituto de Humanidades, Artes re Ciências Professor Milton Santos, Universidade Federal da Bahia, Salvador, Brasil. ${ }_{6}$ Departamento de Saúde Comunitária, Universidade Federal do Ceará, Fortaleza, Brasil.

7 Departamento de Saúde Coletiva, Universidade Federal de Goiás, Goiânia, Brasil.

8 Departamento de Nutrição, Universidade Federal de Pernambuco, Recife, Brasil.

${ }^{9}$ Fundação Maria Cecilia Souto Vidigal, São Paulo, Brasil.

10 Itaú Social, São Paulo, Brasil. 


\section{Introdução}

O desenvolvimento infantil abrange uma série de capacidades e habilidades inter-relacionadas e interdependentes. As principais características nesta perspectiva incluem a linguagem (expressiva e receptiva), as habilidades motoras (amplas e finas), capacidades cognitivas e aspectos socioemocionais da criança 1. Quando as condições socioeconômicas, nutricionais, de estimulação cognitiva, cuidados na primeira infância e de saúde infantil e materna são adequadas para o desenvolvimento da criança, estas capacidades e habilidades tendem a se manifestar de forma similar em diferentes contextos socioculturais 2,3. O estudo Fetal Growth Longitudinal Study do Projeto INTERGROWTH-21st forneceu evidências de que, em populações saudáveis, isto é, com adequada saúde, nutrição e escolaridade maternas, o crescimento e desenvolvimento do encéfalo durante o período gestacional foi similar nos diferentes países (Brasil, Índia, Itália, Quênia e Reino Unido) 4. Análises do mesmo estudo indicaram que, em crianças de famílias com condições socioeconômicas, ambientais e assistenciais adequadas, o crescimento e neurodesenvolvimento até os dois anos de idade foram também similares nesses países 3 .

Existe um conjunto de evidências sobre fatores de risco contextuais individuais/biológicos e/ou psicossociais precoces associados aos déficits ou atrasos no desenvolvimento infantil 5,6. Recentemente, a Organização Mundial da Saúde (OMS) propôs um modelo dos determinantes relacionados a nutrição e cuidados para adequado desenvolvimento, desde a preconcepção até a adolescência $6 . \mathrm{O}$ déficit nutricional de ácido fólico materno, durante o período preconcepcional, está associado à ocorrência de má formação do tubo neural 7 e, consequentemente, a prejuízo no adequado desenvolvimento do sistema nervoso fetal. Durante o período gestacional, a inadequada nutrição materna pode determinar problemas no desenvolvimento e de saúde ao longo do ciclo vital 8,9. Há evidência de que crianças nascidas prematuramente ou pequenas para a idade gestacional (PIG) também apresentam maior risco para atraso no desenvolvimento 6 . Nos últimos anos, há crescente evidência da importância de fatores psicossociais sobre o desenvolvimento infantil 10. A qualidade do cuidado direcionado à criança, por meio de práticas parentais responsivas, afetivas e não punitivas, é importante para o adequado desenvolvimento infantil e capital humano 6,10.

Uma revisão sistemática da literatura identificou que filhos de mães com baixa escolaridade e expostos a condições precárias de saneamento apresentaram piores escores de desenvolvimento cognitivo e motor, em comparação a seus pares, nascidos em famílias com melhores condições socioeconômicas ${ }^{11}$. Da mesma forma, crianças nascidas pré-termo apresentam maior risco para problemas de desenvolvimento quando comparadas àquelas nascidas a termo ${ }^{11}$, e déficits de comprimento para a idade, nos primeiros dois anos de vida, estão associados ao pior desenvolvimento cognitivo na infância e pré-adolescência 12 .

Neste contexto, procurou-se avaliar os fatores socioeconômicos, familiares e individuais associados ao desenvolvimento infantil (linguagem, coordenação motora ampla/fina, pessoal-social e resolução de problemas) durante o primeiro ano de vida de crianças brasileiras em situação de vulnerabilidade social.

\section{Métodos}

\section{Desenho do estudo}

Trata-se de uma análise transversal com dados da linha de base do estudo Avaliação do Impacto do Programa Criança Feliz 13.

\section{Participantes}

Participaram do estudo as mães ( $\pm 99 \%$ das respondentes) ou cuidadores/responsáveis por crianças menores de 12 meses de idade, beneficiárias do Programa Bolsa Família e elegíveis para o Programa Criança Feliz. Estas mães residiam em 30 municípios brasileiros (média de 109 famílias por município), distribuídos em seis estados da Federação. Em cada estado, foram selecionados entre três e seis municípios. 


\section{Amostragem}

A escolha de estados e municípios foi intencional, tendo como base a implementação do Programa Criança Feliz. A amostra foi selecionada a partir da identificação de crianças elegíveis para o Programa Criança Feliz residentes na zona urbana do município. Após aceite em participar do estudo, uma planilha com a lista de crianças elegíveis em cada município foi elaborada e com base nesta planilha foram sorteadas as crianças em cada grupo. O cálculo do tamanho da amostra considerou erro alfa bicaudal de $5 \%$, erro beta de $10 \%$, adesão ao programa de $60 \%$ e acréscimo de $20 \%$ para perdas e recusas. Desta forma, seriam necessárias, em média, 96 crianças por município, totalizando 2.880 crianças no país. Foram incluídos estados de todas as regiões do país, exceto da Região Sul, onde a clientela do Bolsa Família é reduzida. A Região Sul não pode ser incorporada ao estudo por apresentar poucos municípios com o número de crianças elegíveis para o programa, assim como pela existência, nos estados que a compõem, de programas similares ao Programa Criança Feliz, o que dificultaria a obtenção de um grupo controle. Com base nos dados do Cadastro Único 14, foram selecionados municípios que atendessem aos seguintes critérios: aqueles que tinham aderido ao Programa Criança Feliz em 2016; população urbana $\geq 10$ mil habitantes; número de crianças $\leq 12$ meses de idade quatro vezes superior à capacidade instalada do Programa Criança Feliz (visitas semanais a pelo menos 80 crianças e suas famílias); e população maior ou igual a 800 crianças $\leq 12$ meses beneficiárias do Programa Bolsa Família. No estudo de avaliação do impacto, a randomização das famílias para os grupos intervenção e controle foi realizada após a coleta de dados da linha de base. A análise atual utilizou dados de toda amostra com informações disponíveis na linha de base, isto é, antes que as crianças do grupo intervenção fossem incluídas no Programa Criança Feliz. Maiores detalhes sobre o processo de amostragem estão disponíveis em publicação anterior 13 .

\section{Coleta de dados}

Em cada estado, equipes de pesquisa visitaram os domicílios das famílias selecionadas. Os questionários foram aplicados nos domicílios às mães ou responsáveis por entrevistadoras treinadas para aplicação padronizada do instrumento.

\section{Avaliação do desenvolvimento infantil}

O principal desfecho deste estudo foi a avaliação do desenvolvimento infantil, por meio do Ages and Stages Questionnaire (ASQ-3) 15,16,17. O ASQ-3 é um instrumento válido e confiável de avaliação e monitoramento do desenvolvimento da cognição (resolução de problemas), comunicação, desenvolvimento psicomotor (coordenação ampla e fina) e social-pessoal de crianças entre 0 e 6 anos 15,16,17. No Brasil, o estudo de validação psicométrica incluiu \pm 45 mil crianças das diferentes faixas etárias. Nas crianças < 14 meses, a consistência interna (alfa de Cronbach) variou de 0,67-0,75 para o domínio de comunicação, de 0,71-0,85 para coordenação motora ampla, 0,77-0,79 para coordenação motora fina, 0,72-0,79 para resolução de problemas e 0,58-0,76 para o domínio pessoal-social 15. Análises Rasch, seguindo o modelo da Teoria de Resposta ao Item, indicaram que $<10 \%$ dos itens avaliados apresentaram algum problema de ajuste entre os 540 itens avaliados nas diferentes faixas etárias 15 .

Para avaliação de crianças no primeiro ano de vida, o ASQ-3 é composto por seis questionários, aplicados às faixas etárias de 2, 4, 6, 8, 10 e 12 meses. Cada questionário avalia crianças de \pm 1 mês em relação à idade a que se destina. Por exemplo, o questionário de 2 meses inclui crianças com idade de 1 mês e 0 dias até 2 meses e 30 dias. Na aplicação do ASQ-3 em crianças prematuras, seleciona-se o questionário apropriado à faixa etária, ajustando para a prematuridade. $\mathrm{O}$ instrumento avalia os marcos do desenvolvimento (cognição, comunicação, psicomotor e social-pessoal) de acordo com a faixa etária da criança, com itens ordenados de mais simples a mais complexos. O domínio de comunicação avalia, entre outras coisas, a produção de sons, palavras e expressão facial. A coordenação motora ampla avalia o controle motor dos membros superiores e inferiores, controle do tronco, equilíbrio etc. A coordenação motora fina é avaliada por questões sobre segurar e agarrar objetos e interação com objetos (brinquedos, pessoas etc.). O domínio sobre "resolução de problemas" avalia o contato visual (seguir objetos visualmente) e interação com o ambiente (busca estímulos). As perguntas sobre a área 
"pessoal/social" incluem a resposta a reflexos, sorriso social, controle de objetos para alimentação e interação com cuidadores.

Cada domínio do ASQ-3 é composto por seis questões. Cada questão possui três opções de resposta, que indicam o alcance de cada habilidade pela criança ( 0 pontos = ainda não; 5 pontos = às vezes e 10 pontos $=$ sim). $\mathrm{O}$ somatório das respostas compõe o escore de cada domínio considerando a faixa etária da criança. O somatório de todos os domínios foi analisado como escore total do ASQ-3. O tempo médio de aplicação do ASQ-3 foi de 10 minutos.

\section{Fatores de risco investigados}

\section{- Características da família}

(1) Número de crianças menores de sete anos residentes no domicílio (1, 2, 3 ou mais);

(2) Idade paterna em anos $(<20,20-29,30-39,40-49,50-59, \geq 60)$.

\section{- Características sobre a mãe/cuidador principal da criança}

(1) Idade em anos (<15, 15-19, 20-24, 25-29, 30-34, 35-39 e $\geq 40)$;

(2) Cor/raça autodeclarada, de acordo com a classificação do Instituto Brasileiro de Geografia e Estatística (categorizada em branca, parda, preta e outras);

(3) Escolaridade em anos completos (0-4, 5-8 e $\geq 9)$;

(4) Residir com marido/companheiro (sim/não);

(5) Trabalho fora de casa (não, alguns dias, todos os dias);

(6) Sintomas depressivos maternos (sim/não), avaliados pela Escala de Depressão de Edimburgo (Edinburgh Postnatal Depression Scale - EPDS), aplicada verbalmente pela entrevistadora seguindo a ordem das perguntas do instrumento. A EPDS é validada para a população brasileira, tanto em mulheres no período pós-parto 18 quanto para uso na população geral 19 e avalia os sintomas referentes aos sete dias anteriores à entrevista. Utilizou-se o ponto de corte $\geq 10$ para definição de presença sintomas de depressão - sensibilidade de 82,7\% (intervalo de 95\% de confiança - IC95\%: 7,0-89,4) e especificidade de 65,3\% (IC95\%: 59,4-71,0) 18 .

\section{- Características do pré-natal e gestação}

(1) Número de consultas pré-natal (categorizado em 0-5 ou $\geq 6$ );

(2) Trimestre de início das consultas pré-natais (primeiro, segundo ou terceiro);

(3) Planejamento da gravidez ( $\operatorname{sim} /$ não);

(4) Autopercepção de apoio/ajuda do pai criança (sim/não);

(5) Autopercepção de apoio/ajuda da família durante a gravidez (sim/não);

(6) Crescimento intrauterino: crianças nascidas com peso abaixo do percentil 10 para o sexo e idade gestacional, conforme a curva padrão definida pelo Projeto INTERGROWTH-21st 20, foram consideradas com restrição do crescimento intrauterino (RCIU) (pequenas para a idade gestacional - PIG). Conforme o crescimento intrauterino, as crianças foram classificadas como "a termo ( $\geq 37$ semanas de idade gestacional) com peso adequado para idade gestacional (AIG)", "pré-termo (< 37 semanas) com peso AIG”, “a termo PIG” e “pré-termo PIG” 20,21.

\section{Treinamento e coleta de dados}

Os entrevistadores foram treinados por 40 horas por meio de apresentação do instrumento, role plays e instruções para solução de problemas de campo. A coleta de dados ocorreu entre agosto de 2018 e julho de 2019, por meio de tablets, em formato eletrônico, utilizando-se a plataforma REDCap 22. Em cada estado, a coleta de dados ocorreu de forma independente, seguindo o protocolo do estudo, o qual, juntamente com os instrumentos utilizados na pesquisa, estão disponíveis online 13. 


\section{Análises estatísticas}

Todas as análises foram realizadas utilizando modelo multinível, no Stata software, versão 13.1 (https://www.stata.com), considerando três níveis: estados, municípios e características familiares e da criança. Os coeficientes de correlação intraclasse e erros-padrão foram estimados conforme características socioeconômicas, familiares e individuais. Pela análise descritiva e univariada, foram obtidas as frequências absolutas/relativas ou a média das variáveis, com IC95\%. A análise multivariada foi realizada utilizando-se duas abordagens: um modelo mínimo, definido com o auxílio de um directed acyclic graph (DAG; Figura 1) 23; e um modelo hierárquico conceitual, definido pelos autores ${ }^{24}$. Este último modelo classifica os determinantes em níveis hierárquicos de causalidade (determinantes distais, intermediários e proximais), em que o efeito de cada variável é ajustado para potenciais fatores de confusão pertencentes ao mesmo nível e a níveis superiores. Variáveis com valores de $\mathrm{p}<0,2$ em cada nível foram mantidas no modelo para ajuste dos níveis seguintes. O nível 1 foi composto por variáveis socioeconômicas e demográficas maternas (escolaridade do pai da criança; e idade, cor/raça e escolaridade maternas). O nível 2 incluiu as variáveis relacionadas à gestação (planejamento da gravidez, apoio do pai da criança e apoio da família). O nível 3 incluiu as variáveis relacionadas ao pré-natal (número de consultas e trimestre da gestação que iniciou o acompanhamento). No nível 4, foi incluída a variável crescimento intrauterino. No nível 5, entraram as variáveis de características no momento da entrevista (residir com marido/companheiro, trabalho materno fora de casa e número de crianças menores de 7 anos no domicílio). Por fim, no nível 6, entrou a variável de sintomas de depressão materna.

\section{Figura 1}

Diagrama causal (directed acyclic graph - DAG) para associação entre determinantes e desenvolvimento infantil (ASQ-3). Programa Criança Feliz, Brasil, 2018.

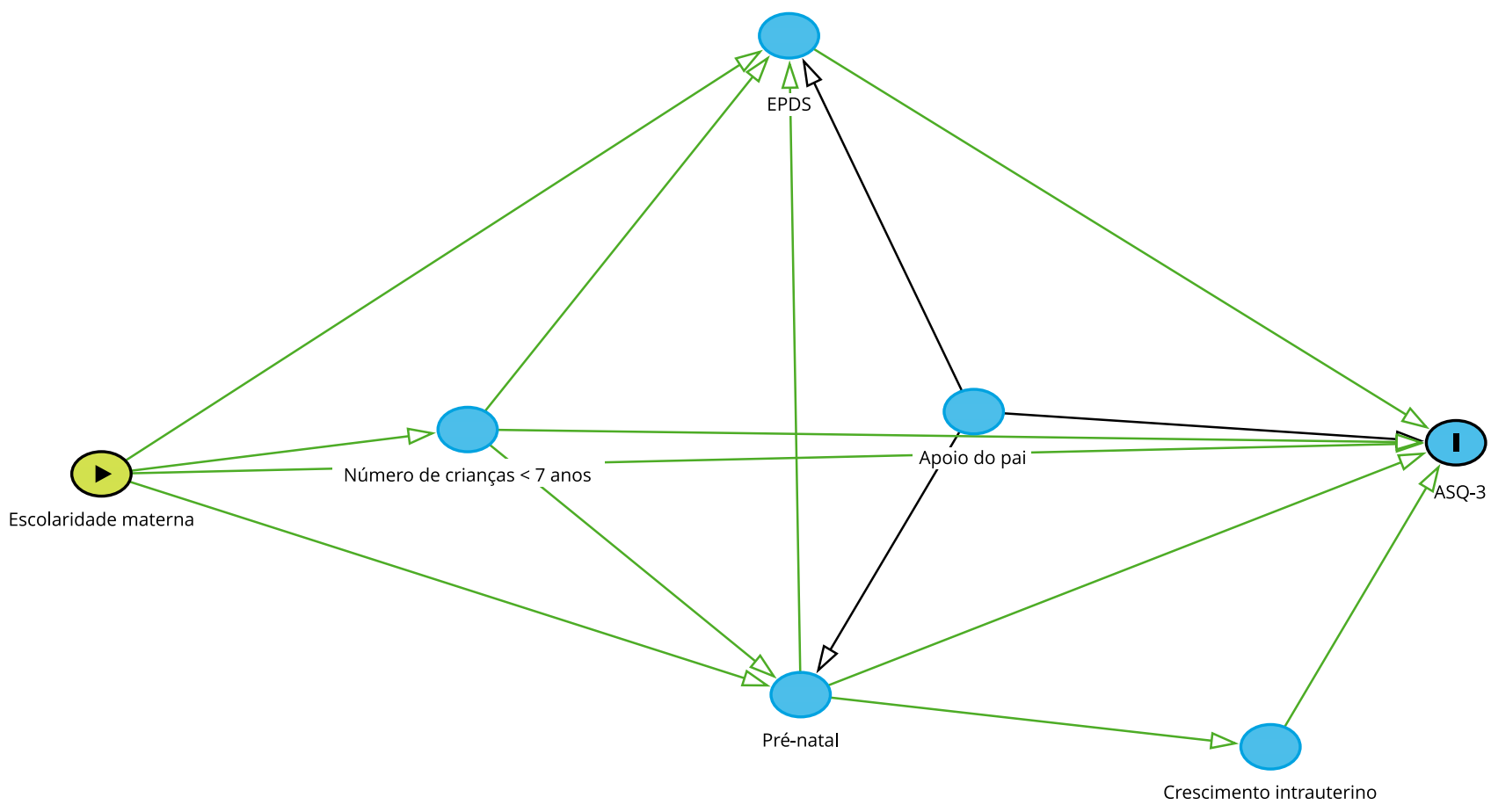

ASQ-3: Ages and Stages Questionnaire; EPDS: Escala de Depressão de Edimburgo. 
$\mathrm{O}$ teste de Wald para heterogeneidade e tendência linear foi usado para avaliar as associações. Valores de p inferiores a 0,05 foram considerados estatisticamente significantes.

\section{Aspectos éticos}

O projeto foi aprovado pelo Comitê de Ética em Pesquisa da Faculdade de Medicina da Universidade Federal de Pelotas (parecer no 2.148.689). O ensaio randomizado está registrado no site do Registro Brasileiro de Ensaios Clínicos (ReBEC; https://ensaiosclinicos.gov.br/), sob o identificador RBR $-4 \times 7$ dny.

\section{Resultados}

Foram analisadas informações de 3.061 crianças (94\% da amostra original), com informações completas para o ASQ-3. Foram excluídas aquelas cujas mães relataram a presença de alguma deficiência $(\mathrm{n}=35)$.

A Tabela 1 apresenta a descrição da amostra incluída nas análises, bem como comparação das frequências relativas com relação ao total de crianças na linha de base do estudo original. Entre todos os determinantes avaliados, apenas a distribuição quanto ao nível socioeconômico da família, à idade e cor/raça maternas apresentaram diferença estatisticamente significantes. Houve maiores perdas entre as crianças de famílias do quintil mais pobre, filhas de mães mais jovens ( $<15$ anos) e que se autodeclararam pardas (Tabela 1).

\section{Tabela 1}

Descrição da amostra ( $N$ = 3.061) conforme características socioeconômicas, familiares e individuais. Programa Criança Feliz, Brasil, 2018.

\begin{tabular}{|c|c|c|c|c|}
\hline \multirow[t]{2}{*}{ Variáveis } & \multicolumn{2}{|c|}{ Distribuição da amostra } & \multirow{2}{*}{$\begin{array}{c}\text { Crianças incluídas } \\
\text { nas análises * } \\
\%\end{array}$} & \multirow[t]{2}{*}{ Valor de $p$ ** } \\
\hline & $\mathbf{n}$ & $\%$ & & \\
\hline Escolaridade do pai (anos) & & & & 0,721 \\
\hline $0-4$ & 431 & 16,8 & 94,3 & \\
\hline $5-8$ & 785 & 30,6 & 93,8 & \\
\hline$\geq 9$ & 1.350 & 52,6 & 94,6 & \\
\hline Idade materna (anos) & & & & 0,042 \\
\hline$<15$ & 38 & 1,3 & 86,4 & \\
\hline $15-19$ & 394 & 13,0 & 93,8 & \\
\hline $20-24$ & 842 & 27,9 & 93,5 & \\
\hline $25-29$ & 707 & 23,4 & 94,5 & \\
\hline $30-34$ & 606 & 20,1 & 95,4 & \\
\hline $35-39$ & 326 & 10,8 & 95,9 & \\
\hline$\geq 40$ & 109 & 3,6 & 98,2 & \\
\hline Cor/Raça materna & & & & 0,043 \\
\hline Branca & 443 & 14,7 & 95,7 & \\
\hline Preta & 310 & 10,3 & 96,6 & \\
\hline Parda & 2.164 & 71,9 & 93,8 & \\
\hline Outras & 93 & 3,1 & 97,9 & \\
\hline
\end{tabular}

(continua) 
Tabela 1 (continuação)

\begin{tabular}{|c|c|c|c|c|}
\hline \multirow[t]{2}{*}{ Variáveis } & \multicolumn{2}{|c|}{ Distribuição da amostra } & \multirow{2}{*}{$\begin{array}{c}\text { Crianças incluídas } \\
\text { nas análises * } \\
\%\end{array}$} & \multirow[t]{2}{*}{ Valor de $p$ ** } \\
\hline & $\mathbf{n}$ & $\%$ & & \\
\hline Escolaridade materna (anos) & & & & 0,525 \\
\hline $0-4$ & 258 & 9,1 & 92,8 & \\
\hline $5-8$ & 873 & 30,8 & 94,5 & \\
\hline$\geq 9$ & 1.702 & 60,1 & 94,5 & \\
\hline Gravidez planejada & & & & 0,482 \\
\hline Não & 2.116 & 70,1 & 94,7 & \\
\hline $\operatorname{Sim}$ & 902 & 29,9 & 94,1 & \\
\hline Apoio do pai durante a gravidez & & & & 0,508 \\
\hline Não & 433 & 14,4 & 95,2 & \\
\hline $\operatorname{Sim}$ & 2.580 & 85,6 & 94,4 & \\
\hline Apoio da família durante a gravidez & & & & 0,406 \\
\hline Não & 221 & 7,4 & 95,7 & \\
\hline Sim & 2.784 & 92,6 & 94,4 & \\
\hline Número de consultas pré-natais & & & & 0,107 \\
\hline$\geq 6$ & 2.457 & 81,4 & 94,2 & \\
\hline $0-5$ & 560 & 18,6 & 95,9 & \\
\hline Trimestre de início pré-natal & & & & 0,945 \\
\hline Primeiro & 2.327 & 77,1 & 94,5 & \\
\hline Segundo & 527 & 17,5 & 94,8 & \\
\hline Terceiro & 163 & 5,4 & 94,2 & \\
\hline Crescimento intrauterino & & & & 0,813 \\
\hline A termo (AIG) & 2.318 & 84,5 & 94,4 & \\
\hline Pré-termo (AIG) & 221 & 8,1 & 94,8 & \\
\hline A termo (PIG) & 191 & 6,9 & 94,1 & \\
\hline Pré-termo (PIG) & 14 & 0,5 & 100,0 & \\
\hline Vive com marido ou companheiro & & & & 0,059 \\
\hline Não & 1.143 & 37,4 & 95,4 & \\
\hline $\operatorname{Sim}$ & 1.917 & 62,6 & 93,8 & \\
\hline Trabalha fora de casa & & & & 0,157 \\
\hline Não & 2.722 & 90,1 & 94,2 & \\
\hline Todos os dias & 120 & 4,0 & 97,6 & \\
\hline Alguns dias & 179 & 5,9 & 96,2 & \\
\hline Número de crianças $<7$ anos & & & & 0,091 \\
\hline 1 & 1.507 & 49,8 & 95,1 & \\
\hline$\geq 2$ & 1.521 & 50,2 & 93,8 & \\
\hline Depressão materna & & & & 0,280 \\
\hline$(E P D S \geq 10)$ & & & & \\
\hline Não & 2.202 & 73,3 & 94,4 & \\
\hline Sim & 802 & 26,7 & 95,4 & \\
\hline
\end{tabular}

AIG: adequadas para idade gestacional; ASQ-3: Ages and Stages Questionnaire; EPDS: Escala de Depressão de Edimburgo; PIG: pequenas para a idade gestacional.

Nota: $\mathrm{N}$ total da variável (diferenças no total amostral são referentes a falta de informação).

* Proporção de crianças incluídas em relação à amostra total do estudo $(n=3.242)$;

** Teste estatístico (qui-quadrado) para diferença entre amostra incluída no estudo e amostra com informações para ASQ-3 total e domínios. 
A Tabela 2 apresenta a média e IC95\% do escore do ASQ-3 total e por domínio, de acordo com os potenciais determinantes do desenvolvimento infantil (análise bivariada). Sete das 15 variáveis estudadas associaram-se $(\mathrm{p}<0,05)$ com o escore total do ASQ-3: observaram-se menores escores entre crianças pertencentes a famílias com maior número de crianças menores de 7 anos, cujas mães tinham menor escolaridade, sintomas depressivos, com menor número e início tardio (terceiro trimestre) de consultas pré-natais, que não receberam apoio do pai da criança durante a gestação, e as prematuras PIG (Tabela 2). Apenas crescimento intrauterino, baixa escolaridade materna e sintomas depressivos maternos associaram-se com todos os cinco domínios do teste. Já o apoio do pai da criança durante a gestação e número de menores de sete anos na casa associaram-se a quatro domínios do ASQ-3. O domínio mais fortemente associado com fatores de risco foi o da comunicação (associado a 8 dos 15 fatores), enquanto os domínios coordenação motora ampla e pessoal-social apresentaram o menor número de associações (5 fatores).

Tabela 2

Análise multinível não ajustada, médias, intervalo de 95\% de confiança (IC95\%) do ASQ-3 (total e por domínio) segundo fatores socioeconômicos, familiares e individuais. Programa Criança Feliz, Brasil, 2018.

\begin{tabular}{|c|c|c|c|c|c|c|}
\hline Variáveis & Média (IC95\%) & Média (IC95\%) & $\begin{array}{l}\text { Coordenação } \\
\text { motora ampla } \\
\text { Média (IC95\%) }\end{array}$ & $\begin{array}{l}\text { Coordenação } \\
\text { motora fina } \\
\text { Média (IC95\%) }\end{array}$ & $\begin{array}{l}\text { Resolução de } \\
\text { problemas } \\
\text { Média (IC95\%) }\end{array}$ & Pessoal-social \\
\hline \multicolumn{7}{|l|}{ Nível 1} \\
\hline $\begin{array}{l}\text { Escolaridade do } \\
\text { pai (anos) }\end{array}$ & $p=0,141$ & $p=0,015$ & $p=0,506$ & $p=0,155$ & $p=0,930$ & $p=0,105$ \\
\hline $0-4$ & $247,2(243,1 ; 251,3)$ & $49,6(48,6 ; 50,5)$ & $48,0(46,5 ; 49,5)$ & $51,2(50,1 ; 52,4)$ & $50,2(49,0 ; 51,5)$ & $48,0(46,9 ; 49,1)$ \\
\hline $5-8$ & $247,8(244,5 ; 251,1)$ & $50,2(49,5 ; 50,8)$ & $48,7(47,4 ; 50,0)$ & $50,6(49,7 ; 51,5)$ & $50,3(49,4 ; 51,3)$ & $48,0(47,2 ; 48,9)$ \\
\hline$\geq 9$ & $250,6(247,8 ; 253,4)$ & $50,8(50,1 ; 51,5)$ & $48,8(47,7 ; 50,0)$ & $51,5(50,8 ; 52,3)$ & $50,5(49,7 ; 51,2)$ & $49,0(48,3 ; 49,6)$ \\
\hline $\begin{array}{l}\text { Idade materna } \\
\text { (anos) }\end{array}$ & $p=0,256$ & $p=0,863$ & $p=0,306$ & $p=0,721$ & $p=0,108$ & $p=0,501$ \\
\hline$<15$ & $249,7(245,8 ; 253,6)$ & $50,2(49,2 ; 51,2)$ & $46,0(41,9 ; 50,0)$ & $49,2(45,7 ; 52,7)$ & $49,7(45,8 ; 53,6)$ & $47,6(43,9 ; 51,2)$ \\
\hline $15-19$ & $249,1(246,0 ; 252,3)$ & $50,2(49,4 ; 51,0)$ & $49,2(47,6 ; 50,8)$ & $50,9(49,7 ; 52,1)$ & $51,3(49,9 ; 52,6)$ & $48,6(47,4 ; 49,8)$ \\
\hline $20-24$ & $248,5(245,9 ; 251,2)$ & $50,2(49,5 ; 50,9)$ & $49,0(47,6 ; 50,3)$ & $51,2(50,3 ; 52,0)$ & $50,8(49,7 ; 51,9)$ & $48,4(47,5 ; 49,2)$ \\
\hline $25-29$ & $248,0(245,5 ; 250,5)$ & $50,2(49,6 ; 50,8)$ & $48,4(47,0 ; 49,8)$ & $50,7(49,8 ; 51,6)$ & $49,4(48,2 ; 50,5)$ & $47,3(46,4 ; 48,3)$ \\
\hline $30-34$ & $247,4(244,7 ; 250,1)$ & $50,2(49,5 ; 50,9)$ & $47,9(46,5 ; 49,3)$ & $51,1(50,1 ; 52,1)$ & $49,7(48,5 ; 50,9)$ & $48,3(47,3 ; 49,4)$ \\
\hline $35-39$ & $246,8(243,5 ; 250,0)$ & $50,2(49,3 ; 51,0)$ & $48,0(46,3 ; 49,7)$ & $51,4(50,1 ; 52,7)$ & $50,3(48,8 ; 51,8)$ & $48,5(47,2 ; 49,8)$ \\
\hline$\geq 40$ & $246,2(242,2 ; 250,2)$ & $50,1(49,1 ; 51,2)$ & $47,1(44,6 ; 49,6)$ & $52,3(50,2 ; 54,4)$ & $48,9(46,5 ; 51,3)$ & $47,4(45,2 ; 49,6)$ \\
\hline Cor/Raça materna & $p=0,311$ & $p=0,797$ & $p=0,357$ & $p=0,557$ & $p=0,297$ & $p=0,340$ \\
\hline Branca & $246,5(242,6 ; 250,5)$ & $50,2(49,2 ; 51,2)$ & $47,6(46,0 ; 49,1)$ & $51,0(49,9 ; 52,1)$ & $50,6(49,2 ; 51,9)$ & $48,0(46,8 ; 49,1)$ \\
\hline Preta & $247,5(244,7 ; 250,3)$ & $50,2(49,5 ; 50,9)$ & $48,0(46,3 ; 49,8)$ & $50,3(49,0 ; 51,6)$ & $48,9(47,4 ; 50,5)$ & $47,4(46,0 ; 48,7)$ \\
\hline Parda & $248,4(245,8 ; 251,1)$ & $50,2(49,6 ; 50,9)$ & $48,7(47,5 ; 49,8)$ & $51,2(50,6 ; 51,8)$ & $50,3(49,5 ; 51,2)$ & $48,3(47,7 ; 49,0)$ \\
\hline Outras & $249,4(245,8 ; 253,0)$ & $50,2(49,3 ; 51,2)$ & $48,9(46,2 ; 51,6)$ & $51,8(49,5 ; 54,0)$ & $50,0(47,4 ; 52,5)$ & $46,8(44,4 ; 49,1)$ \\
\hline \multicolumn{7}{|l|}{ materna (anos) } \\
\hline $0-4$ & $239,1(235,0 ; 243,2)$ & $48,7(47,6 ; 49,8)$ & $46,3(44,4 ; 48,1)$ & $49,1(47,7 ; 50,5)$ & $48,0(46,4 ; 49,6)$ & $45,9(44,4 ; 47,3)$ \\
\hline $5-8$ & $245,0(242,3 ; 247,7)$ & $49,7(49,0 ; 50,4)$ & $47,9(46,5 ; 49,2)$ & $50,5(49,7 ; 51,4)$ & $49,6(48,6 ; 50,6)$ & $47,9(47,0 ; 48,8)$ \\
\hline$\geq 9$ & $251,0(248,3 ; 253,6)$ & $50,7(50,0 ; 51,4)$ & $49,0(47,8 ; 50,2)$ & $51,7(51,1 ; 52,4)$ & $50,8(49,9 ; 51,6)$ & $48,5(47,8 ; 49,2)$ \\
\hline \multicolumn{7}{|l|}{ Nível 2} \\
\hline Gravidez planejada & $p=0,752$ & $p=0,613$ & $p=0,749$ & $p=0,374$ & $p=0,567$ & $p=0,374$ \\
\hline Não & $247,8(245,2 ; 250,4)$ & $50,2(49,5 ; 50,8)$ & $48,5(47,3 ; 49,6)$ & $50,9(50,3 ; 51,5)$ & $50,3(49,4 ; 51,1)$ & $48,0(47,3 ; 48,6)$ \\
\hline Sim & $248,3(245,0 ; 251,6)$ & $50,2(49,4 ; 51,1)$ & $48,3(47,0 ; 49,7)$ & $51,3(50,5 ; 52,1)$ & $50,0(48,9 ; 51,0)$ & $48,4(47,5 ; 49,3)$ \\
\hline
\end{tabular}

(continua) 
Tabela 2 (continuação)

\begin{tabular}{|c|c|c|c|c|c|c|}
\hline Variáveis & Média (IC95\%) & Média (IC95\%) & $\begin{array}{l}\text { Coordenação } \\
\text { motora ampla } \\
\text { Média (IC95\%) }\end{array}$ & $\begin{array}{l}\text { Coordenação } \\
\text { motora fina } \\
\text { Média (IC95\%) }\end{array}$ & $\begin{array}{l}\text { Resolução de } \\
\text { problemas } \\
\text { Média (IC95\%) }\end{array}$ & Média (IC95\%) \\
\hline $\begin{array}{l}\text { Apoio do pai } \\
\text { durante a gravidez }\end{array}$ & $\mathrm{p}<0,001$ & $p=0,053$ & $p=0,002$ & $p=0,001$ & $p=0,008$ & $p=0,036$ \\
\hline Não & $241,4(237,2 ; 245,6)$ & $49,5(48,4 ; 50,6)$ & $46,8(45,2 ; 48,3)$ & $49,5(48,4 ; 50,6)$ & $48,7(47,3 ; 50,1)$ & $47,0(45,9 ; 48,2)$ \\
\hline Sim & $249,1(246,5 ; 251,7)$ & $50,3(49,7 ; 51,0)$ & $48,7(47,5 ; 49,9)$ & $51,3(50,7 ; 51,9)$ & $50,4(49,6 ; 51,3)$ & $48,3(47,7 ; 48,9)$ \\
\hline $\begin{array}{l}\text { Apoio da família } \\
\text { durante a gravidez }\end{array}$ & $p=0,081$ & $p=0,005$ & $p=0,151$ & $p=0,149$ & $p=0,934$ & $p=0,595$ \\
\hline Não & $243,7(238,2 ; 249,2)$ & $48,4(47,0 ; 49,8)$ & $47,3(45,4 ; 49,2)$ & $50,0(48,5 ; 51,5)$ & $50,1(48,4 ; 51,9)$ & $47,7(46,1 ; 49,3)$ \\
\hline Sim & $248,4(246,0 ; 250,8)$ & $50,4(49,7 ; 51,0)$ & $48,5(47,4 ; 49,7)$ & $51,2(50,6 ; 51,7)$ & $50,2(49,4 ; 51,0)$ & $48,2(47,6 ; 48,7)$ \\
\hline $\begin{array}{l}\text { Nível } 3 \\
\text { Número de } \\
\text { consultas pré- } \\
\text {-natais }\end{array}$ & $p=0,013$ & $p=0,001$ & $p=0,036$ & $p=0,093$ & $p=0,176$ & $p=0,761$ \\
\hline $\begin{array}{l}\geq 6 \\
0-5\end{array}$ & $\begin{array}{l}248,8(246,5 ; 251,2) \\
244,3(240,6 ; 248,0)\end{array}$ & $\begin{array}{l}50,5(49,8 ; 51,1) \\
49,0(48,0 ; 50,0)\end{array}$ & $\begin{array}{l}48,7(47,6 ; 49,8) \\
47,4(46,0 ; 48,9)\end{array}$ & $\begin{array}{l}51,2(50,7 ; 51,8) \\
50,3(49,3 ; 51,3)\end{array}$ & $\begin{array}{l}50,3(49,5 ; 51,2) \\
49,5(48,3 ; 50,8)\end{array}$ & $\begin{array}{l}48,1(47,5 ; 48,7) \\
48,0(46,9 ; 49,0)\end{array}$ \\
\hline $\begin{array}{l}\text { Trimestre de início } \\
\text { do pré-natal }\end{array}$ & $p=0,004$ & $p=0,012$ & $p=0,193$ & $p=0,237$ & $p=0,024$ & $p=0,198$ \\
\hline Primeiro & $249,0(246,5 ; 251,5)$ & $50,4(49,8 ; 51,1)$ & $48,6(47,5 ; 49,8)$ & $51,2(50,6 ; 51,8)$ & $50,4(49,6 ; 51,3)$ & $48,2(47,6 ; 48,8)$ \\
\hline Segundo & $245,4(242,4 ; 248,3)$ & $49,5(48,8 ; 50,3)$ & $47,9(46,7 ; 49,1)$ & $50,6(49,9 ; 51,4)$ & $49,5(48,5 ; 50,5)$ & $48,2(47,2 ; 49,3)$ \\
\hline Terceiro & $241,7(236,9 ; 246,6)$ & $48,7(47,3 ; 50,0)$ & $47,2(45,4 ; 48,9)$ & $50,1(48,7 ; 51,4)$ & $48,6(47,0 ; 50,2)$ & $46,5(44,7 ; 48,3)$ \\
\hline $\begin{array}{l}\text { Nível } 4 \\
\text { Crescimento } \\
\text { intrauterino }\end{array}$ & $p=0,001$ & $p=0,037$ & $p<0,001$ & $p=0,003$ & $p<0,001$ & $p<0,001$ \\
\hline A termo (AIG) & $249,4(247,3 ; 251,6)$ & $50,5(50,0 ; 51,0)$ & $49,0(47,9 ; 50,1)$ & $51,2(50,5 ; 51,9)$ & $50,5(49,7 ; 51,3)$ & $48,4(47,9 ; 49,0)$ \\
\hline Pré-termo (AIG) & $245,5(242,7 ; 248,2)$ & $49,9(49,2 ; 50,6)$ & $44,5(42,6 ; 46,4)$ & $49,3(47,7 ; 50,8)$ & $48,1(46,4 ; 49,8)$ & $46,0(44,4 ; 47,5)$ \\
\hline A termo (PIG) & $241,5(236,7 ; 246,3)$ & $49,4(48,1 ; 50,6)$ & $49,1(47,0 ; 51,1)$ & $50,4(48,7 ; 52,1)$ & $50,4(48,5 ; 52,2)$ & $49,3(47,6 ; 51,0)$ \\
\hline Pré-termo (PIG) & $237,5(230,5 ; 244,6)$ & $48,8(47,0 ; 50,7)$ & $41,6(35,2 ; 48,0)$ & $42,8(37,0 ; 48,6)$ & $39,7(33,3 ; 46,0)$ & $39,1(33,2 ; 45,1)$ \\
\hline Nível 5 & & & & & & \\
\hline $\begin{array}{l}\text { Vive com marido } \\
\text { ou companheiro }\end{array}$ & $p=0,248$ & $p=0,102$ & $p=0,445$ & $p=0,055$ & $p=0,961$ & $p=0,966$ \\
\hline Não & $246,8(243,8 ; 249,8)$ & $49,8(49,0 ; 50,6)$ & $48,3(47,0 ; 49,5)$ & $50,5(49,7 ; 51,2)$ & $50,1(49,2 ; 51,1)$ & $48,1(47,3 ; 48,9)$ \\
\hline Sim & $248,5(245,8 ; 251,1)$ & $50,4(49,7 ; 51,1)$ & $48,6(47,4 ; 49,8)$ & $51,3(50,6 ; 51,9)$ & $50,1(49,2 ; 51,0)$ & $48,1(47,4 ; 48,8)$ \\
\hline $\begin{array}{l}\text { Trabalha fora de } \\
\text { casa }\end{array}$ & $p=0,242$ & $p=0,688$ & $p=0,844$ & $p=0,054$ & $p=0,152$ & $p=0,741$ \\
\hline Não & $247,7(245,2 ; 250,3)$ & $50,2(49,6 ; 50,9)$ & $48,4(47,3 ; 49,6)$ & $50,9(50,3 ; 51,5)$ & $50,0(49,2 ; 50,9)$ & $48,1(47,5 ; 48,7)$ \\
\hline Todos os dias & $249,4(246,0 ; 252,8)$ & $50,0(49,1 ; 50,9)$ & $48,5(46,1 ; 51,0)$ & $52,7(50,7 ; 54,7)$ & $50,5(48,2 ; 52,8)$ & $48,7(46,5 ; 50,8)$ \\
\hline Alguns dias & $251,0(245,4 ; 256,7)$ & $49,8(48,3 ; 51,3)$ & $49,0(46,9 ; 51,0)$ & $52,4(50,7 ; 54,0)$ & $51,9(49,9 ; 53,8)$ & $47,6(45,9 ; 49,4)$ \\
\hline $\begin{array}{l}\text { Número de } \\
\text { crianças < } 7 \text { anos }\end{array}$ & $p<0,001$ & $p=0,001$ & $p=0,092$ & $p=0,039$ & $p<0,001$ & $p=0,005$ \\
\hline 1 & $251,1(248,1 ; 254,1)$ & $50,8(50,1 ; 51,6)$ & $48,9(47,7 ; 50,2)$ & $51,4(50,7 ; 52,2)$ & $51,1(50,2 ; 52,1)$ & $48,8(48,0 ; 49,5)$ \\
\hline$\geq 2$ & $245,2(242,3 ; 248,2)$ & $49,6(48,8 ; 50,3)$ & $48,2(46,9 ; 49,4)$ & $50,6(49,9 ; 51,3)$ & $49,3(48,4 ; 50,3)$ & $47,6(46,9 ; 48,3)$ \\
\hline Nível 6 & & & & & & \\
\hline $\begin{array}{l}\text { Depressão } \\
\text { materna (EPDS } \\
\geq 10 \text { ) }\end{array}$ & $p<0,001$ & $\mathrm{p}<0,001$ & $p<0,001$ & $p<0,001$ & $p<0,001$ & $p=0,001$ \\
\hline Não & $250,7(248,0 ; 253,5)$ & $50,6(49,9 ; 51,3)$ & $49,0(47,8 ; 50,1)$ & $51,8(51,2 ; 52,4)$ & $50,8(49,9 ; 51,8)$ & $48,6(47,9 ; 49,2)$ \\
\hline Sim & $240,9(237,5 ; 244,3)$ & $49,2(48,3 ; 50,1)$ & $47,1(45,8 ; 48,4)$ & $49,1(48,2 ; 49,9)$ & $48,6(47,4 ; 49,7)$ & $46,9(46,0 ; 47,8)$ \\
\hline
\end{tabular}

AIG: adequadas para idade gestacional; ASQ-3: Ages and Stages Questionnaire; EPDS: Escala de Depressão de Edimburgo; PIG: pequenas para a idade gestacional. 
A Tabela 3 apresenta resultados das análises de regressão multinível linear bruta e ajustada, considerando os dois modelos de ajuste (DAG e modelo conceitual). Para estas análises, foram incluídas as variáveis que na Tabela 2 apresentaram nível de significância $<0,2$ para o escore total do ASQ-3 (escolaridade paterna e materna, apoio da família e do pai da criança durante a gestação, número e trimestre de início das consultas pré-natais, crescimento intrauterino, número de crianças $<7$ anos e sintomas depressivos maternos).

Tanto a análise com base no DAG, quanto a análise pelo modelo conceitual indicaram associação do escore total do ASQ-3 com escolaridade materna, apoio do pai da criança durante a gestação, crescimento intrauterino, número de crianças $<7$ anos e sintomas depressivos maternos. De maneira geral, em ambos os modelos, os filhos de mães com baixa escolaridade ( 0 - 4 anos) e com sintomas de depressão apresentaram, em média, uma redução de aproximadamente 10 pontos no escore total do ASQ-3. Além disso, a maior redução na média do ASQ-3 total, de aproximadamente 40 pontos (IC95\%: -58,8; -19,6) foi observada entre as crianças nascidas pré-termo PIG. As pertencentes a famílias com duas ou mais crianças $<7$ anos e aquelas cujas mães não receberam apoio do pai da criança durante a gestação apresentaram uma redução aproximada de 5 pontos no escore total médio do ASQ-3 (Tabela 3).

Os coeficientes de correlação intraclasse (ICC) foram apresentados no Material Suplementar (http://cadernos.ensp.fiocruz.br/static//arquivo/suppl-e00316920_9874.pdf). O ICC relata duas correlações intraclasse para este modelo aninhado de três níveis. A primeira coluna (estado) apresenta a correlação intraclasse do nível estadual, isto é, a correlação entre os escores de desenvolvimento no mesmo estado. A segunda coluna (município) apresenta a correlação intraclasse ao nível de município dentro do estado, ou seja, a correlação entre escores de desenvolvimento no mesmo município e estado. Condicionalmente às covariáveis de efeitos fixos, os escores de desenvolvimento apresentaram baixa correlação dentro do mesmo estado (< 1\%), sendo ligeiramente maior dentro do mesmo município e estado $( \pm 1 \%)$. Desta forma, os efeitos aleatórios do município e estado representaram cerca de $1 \%$ da variância residual total em todas as características socioeconômicas, familiares e individuais analisadas.

\section{Discussão}

Os escores de desenvolvimento infantil (total e por domínios) no primeiro ano de vida foram consistentemente menores em filhos de mães com baixa escolaridade, com sintomas de depressão, que não tiveram apoio do pai da criança durante a gestação e com duas ou mais crianças menores de sete anos residindo no domicílio. Prematuridade com RCIU associou-se a menores escores de desenvolvimento para comunicação, coordenação motora ampla e fina, resolução de problemas e pessoal-social.

A associação entre baixa escolaridade materna e redução nos escores de desenvolvimento infantil identificadas neste estudo também foram observadas em outros contextos socioculturais. Uma recente revisão sistemática analisou dados de 21 países de baixa e média renda, incluindo estudos brasileiros, e reportou que a baixa escolaridade materna e paterna, falta de acesso a água potável e saneamento básico, prematuridade, baixo peso ao nascer, baixa estatura materna e anemia na infância associaram-se a menores escores de desenvolvimento em crianças menores de sete anos 11. Por outro lado, a mesma revisão mostrou que a RCIU, duração da amamentação exclusiva, anemia materna e diarreia na criança não apresentavam associação com o desenvolvimento infantil 11. Assim como observado neste estudo, estimativas agrupadas em estudos de metanálise identificaram relação positiva, com gradiente dose-resposta, entre escolaridade dos pais e escores de desenvolvimento infantil ${ }^{11}$. Nilsen et al. 25 realizaram uma metanálise que avaliou dados de 185 estudos. Foram analisados mais de 100 fatores estressores maternos e seus efeitos sobre as habilidades cognitivas em crianças e adolescentes menores de 18 anos. Os estressores socioeconômicos, entre eles a baixa escolaridade materna, foram responsáveis por $13 \%$ e $18 \%$ do atraso de desenvolvimento de habilidades cognitivas em crianças e adolescentes, respectivamente 25 . A literatura sobre os efeitos da escolaridade materna na saúde infantil 26,27,28,29 explica esta associação a partir de mecanismos indiretos, relacionados às inequidades em saúde. Desta forma, grupos populacionais de menor escolaridade e renda têm dificuldades ao acesso e pior qualidade ao cuidado pré-natal, acesso reduzido a condições sanitárias adequadas e menores 
Tabela 3

Regressão multinível linear bruta, ajustada conforme DAG (directed acyclic graph) e modelo hierarquizado conceitual de análise. Programa Criança Feliz, Brasil, 2018.

\begin{tabular}{|c|c|c|c|}
\hline & \multicolumn{3}{|c|}{ ASQ-3 Total (0 a 300 pontos) } \\
\hline & Bruta & DAG * & Modelo conceitual \\
\hline & $\beta($ IC95\%) & $\beta(I C 95 \%)$ & $\beta(I C 95 \%)$ \\
\hline Escolaridade do pai (anos) & $p=0,141$ & $p=0,355$ & $p=0,782$ \\
\hline $0-4$ & $-3,4(-7,6 ; 0,8)$ & $-0,1(-4,6 ; 4,3)$ & $1,6(-2,9 ; 6,1)$ \\
\hline $5-8$ & $-2,8(-6,2 ; 0,6)$ & $-2,5(-6,0 ; 1,0)$ & $0,2(-3,3 ; 3,8)$ \\
\hline$\geq 9$ & 0,0 (referência) & 0,0 (referência) & 0,0 (referência) \\
\hline Escolaridade materna (anos) & $\mathrm{p}<0,001$ & $p=0,001$ & $p=0,010$ \\
\hline $0-4$ & $-12,6(-8,4 ;-2,1)$ & $-10,3(-15,9 ;-4,7)$ & $-8,6(-14,3 ;-2,9)$ \\
\hline $5-8$ & $-5,3(-17,7 ;-7,6)$ & $-2,2(-5,6 ; 1,1)$ & $-2,0(-5,4 ; 1,4)$ \\
\hline$\geq 9$ & 0,0 (referência) & 0,0 (referência) & 0,0 (referência) \\
\hline Apoio do pai durante a gravidez & $p=0,002$ & $p=0,011$ & $p=0,017$ \\
\hline Não & $-7,7(-11,6 ;-3,7)$ & $-5,7(-10,0 ;-1,3)$ & $-5,3(-9,7 ;-0,9)$ \\
\hline $\operatorname{Sim}$ & 0,0 (referência) & 0,0 (referência) & 0,0 (referência) \\
\hline Apoio da família durante a gravidez & $p=0,080$ & $p=0,364$ & $p=0,389$ \\
\hline Não & $4,7(-0,6 ; 10,0)$ & $2,6(-3,0 ; 8,2)$ & $2,4(-3,1 ; 7,9)$ \\
\hline Sim & 0,0 (referência) & 0,0 (referência) & 0,0 (referência) \\
\hline Número de consultas pré-natais & $p=0,013$ & $p=0,557$ & $p=0,775$ \\
\hline$\geq 6$ & 0,0 (referência) & 0,0 (referência) & 0,0 (referência) \\
\hline $0-5$ & $-4,6(-8,2 ;-1,0)$ & $-1,2(-5,2 ; 2,8)$ & $0,6(-3,7 ; 4,9)$ \\
\hline Trimestre de início do pré-natal & $p=0,006$ & $p=0,239$ & $p=0,243$ \\
\hline Primeiro & 0,0 (referência) & 0,0 (referência) & 0,0 (referência) \\
\hline Segundo & $-1,6(-5,3 ; 2,0)$ & $0,6(-3,7 ; 4,8)$ & $-0,7(-5,2 ; 3,8)$ \\
\hline Terceiro & $-10,0(-16,2 ;-3,8)$ & $-5,6(-12,5 ; 1,3)$ & $-6,4(-13,8 ; 1,1)$ \\
\hline Crescimento intrauterino & $p<0,001$ & $p<0,001$ & $p<0,001$ \\
\hline A termo (AIG) & 0,0 (referência) & 0,0 (referência) & 0,0 (referência) \\
\hline Pré-termo (AIG) & $-11,8(-17,1 ;-6,4)$ & $-10,4(-15,9 ;-4,8)$ & $-10,5(-16,0 ;-5,0)$ \\
\hline A termo (PIG) & $-1,0(-6,7 ; 4,6)$ & $-0,4(-6,3 ; 5,5)$ & $-0,7(-6,6 ; 5,2)$ \\
\hline Pré-termo (PIG) & $-37,8(-57,8 ;-17,8$ & $-39,4(-59,0 ;-19,7)$ & $-39,2(-58,8 ;-19,6)$ \\
\hline Número de crianças $<7$ anos & $p<0,001$ & $p=0,002$ & $p=0,004$ \\
\hline 1 & 0,0 (referência) & 0,0 (referência) & 0,0 (referência) \\
\hline$\geq 2$ & $-5,9(-8,6 ;-3,1)$ & $-4,6(-7,6 ;-1,5$ & $-4,5(-7,5 ;-1,4)$ \\
\hline Depressão materna (EPDS $\geq 10$ ) & $p<0,001$ & $p<0,001$ & $p<0,001$ \\
\hline Não & 0,0 (referência) & 0,0 (referência) & 0,0 (referência) \\
\hline Sim & $-9,8(-13,0 ;-6,7)$ & $-8,8(-12,4 ;-5,3)$ & $-8,8(-12,3 ;-5,4)$ \\
\hline
\end{tabular}

AIG: adequadas para idade gestacional; ASQ-3: Ages and Stages Questionnaire; EPDS: Escala de Depressão de Edimburgo; PIG: pequenas para a idade gestacional.

* Variáveis incluídas no modelo (efeito direto): escolaridade e depressão materna, número de crianças < 7 anos, crescimento intrauterino, apoio do pai durante a gravidez e número de consultas pré-natal.

intervalos interpartais, 30 em comparação com aqueles de maior escolaridade. Há evidências de que as práticas de estimulação cognitiva e cuidado responsivo sejam mais frequentes entre as mães/cuidadores mais escolarizados, em comparação a grupos com menor escolaridade 11. Maior escolaridade materna está associada a melhor nutrição materno-infantil 27,30, acesso aos serviços de saúde e maior cobertura vacinal. Dados relacionados a população geral no Brasil, de acordo com a Pesquisa Nacional de Saúde, indicam que dois terços dos indivíduos $(67,1 \%)$ sem escolaridade ou com nível fundamental incompleto tiveram acesso a consulta médica no ano anterior à pesquisa, enquanto esse percentual foi 
de $81 \%$ dos indivíduos com nível superior completo 31. Assim, uma série de mecanismos contextuais e individuais podem explicar a associação entre escolaridade e desenvolvimento infantil. Portanto, estas evidências sugerem que a oferta de ensino formal à população deve estar interrelacionada com políticas e programas da área da saúde.

Os transtornos depressivos são considerados a terceira principal causa de incapacidade no mundo, especialmente entre as mulheres 32 . Há consenso na literatura mundial de que a depressão afeta as mulheres cerca de duas vezes mais do que os homens e que a etiologia do transtorno é multifatorial, com múltiplas combinações e interações entre fatores de risco 33. A depressão materna tem impacto negativo em diferentes aspectos do neurodesenvolvimento e saúde mental de crianças e adolescentes 34,35,36,37,38. Liu et al. 39 conduziram metanálise de 17 estudos para avaliar os efeitos de sintomas depressivos maternos no desenvolvimento cognitivo de crianças menores de sete anos. Sintomas depressivos maternos foram associados a redução de 0,25 desvios padrão (DP) nos escores de desenvolvimento cognitivo dos filhos. Outra revisão sistemática incluiu 73 estudos que avaliaram as consequências da depressão materna no desenvolvimento da linguagem, motor e cognitivo, além de aspectos socioemocionais e de saúde mental das crianças. Quatro em cada 10 estudos reportaram que filhos de mães deprimidas apresentavam menores escores de desenvolvimento motor e de linguagem; dois terços dos estudos identificaram atrasos no desenvolvimento cognitivo em crianças cujas mães eram deprimidas; e 8 em cada 10 estudos reportaram problemas de comportamento e saúde mental como consequências da depressão materna na saúde e desenvolvimento dos filhos 35 . Fatores como baixo nível socioeconômico, menor escolaridade, restrição ao acesso a serviços de saúde e falta de suporte social estão associados a maior ocorrência de depressão em diferentes países do mundo 40 . Além destes fatores, uma explicação plausível para os efeitos da depressão materna no desenvolvimento infantil refere-se aos prejuízos decorrentes da presença do transtorno nas relações e interações familiares, sociais e de trabalho do indivíduo. Há evidências de que mães deprimidas apresentariam pior qualidade e menor quantidade de estímulos sensoriais, cognitivos e afetivos direcionados à criança, em comparação às mães não deprimidas 41 . As práticas parentais (entendidas estratégias e técnicas utilizadas pelos pais e/ou cuidadores para cuidar, educar e possibilitar que os filhos se desenvolvam) tendem a ser mais punitivas e menos afetivas e responsivas nas mães que estão deprimidas 42 . Estudos com filhos de mães deprimidas mostraram padrões de atividade cerebral (registrada em eletroencefalograma) semelhantes aos encontrados em adultos com depressão 43 , sendo este efeito ainda maior para aquelas crianças que passavam ao menos $50 \%$ do tempo sob cuidados das mães com transtorno depressivo 44 . A atividade cerebral em pessoas deprimidas é reduzida e, portanto, a exposição a experiências adversas (como depressão materna) nos primeiros anos de vida pode contribuir para a subespecificação e conexão incorreta dos circuitos cerebrais 45.

Este estudo observou menores escores de desenvolvimento infantil entre crianças nascidas prematuramente e com restrição do crescimento intrauterino. Este resultado também foi observado na metanálise conduzida por Sania et al. 11, citada anteriormente. Os escores padronizados para desenvolvimento cognitivo e motor foram: DP = 0,14 (IC95\%: -0,24; -0,05) e DP = 0,23 (IC95\%: -0,42; -0,03) menores em crianças pré-termo e AIG, em comparação aquelas nascidas a termo e AIG 11. Crianças nascidas pré-termo e PIG também apresentaram uma redução no escore padronizado de desenvolvimento cognitivo (-0,17; IC95\%: -0.29; -0.05). Não foram observadas associações entre prematuridade ou RCIU em relação ao desenvolvimento da linguagem 11. Cabe destacar que outra metanálise, que avaliou resultados de 68 estudos, realizados em 29 países de renda baixa e média, reportou redução nos escores agrupados padronizados e ajustados de desenvolvimento cognitivo e motor em crianças menores de dois anos com déficits de altura para idade 12. Esta possível associação não foi avaliada neste estudo. Destaca-se que o ASQ-3 é ajustado para prematuridade antes da aplicação do instrumento. Desta forma, é plausível supor que o efeito da prematuridade/RCIU não tenha sido superestimado na atual análise. Um ponto forte deste estudo é, além da contribuição para a investigação dos determinantes do desenvolvimento infantil, a mensuração de diferentes aspectos do desenvolvimento. Soma-se a isso o perfil da amostra, composta por famílias em situação de vulnerabilidade social, em trinta municípios, de diferentes regiões do país. A mensuração do desenvolvimento na primeira infância tem sido apontada como um grande desafio para diferentes países do mundo, devido à multiplicidade de instrumentos de avaliação e restrições inerentes a diferentes instrumentos (nível individual vs. nível populacional, por exemplo) 46. O instrumento ASQ-3 avalia os domínios predominantemente 
empregados na literatura internacional (cognitivo, comunicação e motor) 46, tem sido utilizado em diferentes estudos populacionais 47 e apresenta boas propriedades para rastreamento do desenvolvimento na primeira infância 46,47. O teste pode ser aplicado por entrevistadores com escolaridade de segundo grau ou superior, sem a necessidade de formação específica em Psicologia. A robustez dos resultados obtidos e a identificação de fatores de risco compatíveis com a literatura internacional sugerem que o teste foi aplicado de forma adequada em contexto de entrevistas domiciliares.

A legislação do Marco Legal da Primeira Infância 48 estabelece que as políticas públicas deverão monitorar, coletar dados e avaliar periodicamente os elementos que constituem a oferta dos serviços à criança. Assim, a avaliação do desenvolvimento da criança com instrumentos adequados torna-se fundamental para atingir esse objetivo. Este estudo pode ser considerado como um modelo de avaliação do desenvolvimento da criança a ser seguido por políticas públicas. Conhecer as relações entre as características socioeconômicas, familiares e da criança associados ao desenvolvimento infantil, de populações participantes de programas de políticas públicas, poderá auxiliar os gestores na identificação de fatores risco e de proteção para subsidiar as intervenções.

Os resultados destacam a importância dos determinantes socioeconômicos (escolaridade materna), da saúde mental materna e do crescimento intrauterino no desenvolvimento das crianças durante o primeiro ano de vida. Além de fornecerem evidências para as políticas públicas na primeira infância, os resultados reforçam a importância dos determinantes sociais em saúde, uma vez que os determinantes com maior impacto na redução do escore de desenvolvimento, em todos os domínios avaliados (baixa escolaridade e depressão maternas e RCIU), são potencialmente modificáveis. Intervenções em nível individual e populacional necessitam focar nestes grupos para minimizar os impactos adversos destes determinantes no adequado desenvolvimento infantil. A adequação, ampliação e oferta de políticas públicas no setor saúde, educação e proteção social são fundamentais para redução de diferentes desfechos em saúde, incluindo o desenvolvimento infantil. 


\section{Colaboradores}

T. N. Munhoz contribuiu na análise dos dados, concepção do estudo, interpretação dos dados e resultados, na escrita de todas as versões do manuscrito, na revisão crítica e aprovação da versão final do manuscrito. I. S. Santos, C. Blumenberg, R. S. Barcelos, C. C. Bortolotto, A. Matijasevich, H. G. Santos Júnior, L. M. Santos, L. L. Correa, M. R. Souza, P. I. C. Lira, E. R. P. Altafim, E. C. Macana e C. G. Victora contribuíram na concepção do estudo, interpretação dos dados e resultados, na escrita de todas as versões do manuscrito, na revisão crítica e aprovação da versão final do manuscrito.

\section{Informações adicionais}

ORCID: Tiago N. Munhoz (0000-0003-12819542); Iná S. Santos (0000-0003-1258-9249); Cauane Blumenberg (0000-0002-4580-3849); Raquel Siqueira Barcelos (0000-0001-6580-0509); Caroline C. Bortolotto (0000-0003-3318-7900); Alicia Matijasevich (0000-0003-0060-1589); Hernane G. Santos Júnior (0000-0002-9998-2141); Letícia Marques dos Santos (0000-0001-5963-2166); Luciano L. Correa (0000-0001-8948-8660); Marta Rovery de Souza (0000-0001-6910-843X); Pedro I. C. Lira (0000-0002-1534-1620); Elisa Rachel Pisani Altafim (0000-0002-5732-0473); Esmeralda Correa Macana (0000-0003-1899-3250); Cesar G. Victora (0000-0002-2465-2180).

\section{Agradecimentos}

Este artigo foi realizado com dados do estudo Avaliação de Impacto do Programa Criança Feliz, conduzido pelo Programa de Pós-graduação em Epidemiologia da Universidade Federal de Pelotas, em parceria com o Instituto de Humanidades, Artes \& Ciências Professor Milton Santos (Bahia), Universidade Federal do ABC Paulista, Universidade Federal do Ceará, Universidade Federal de Goiás, Universidade Federal do Oeste do Pará, Universidade Federal de Pernambuco e Universidade de São Paulo, com o apoio do Ministério da Cidadania e do Programa das Nações Unidas para o Desenvolvimento. Estudo foi financiado pela Fundação Maria Cecilia Souto Vidigal (FMCSV), Itaú Social, Programa das Nações Unidas para o Desenvolvimento (PNUD) e Ministério da Cidadania. I. S. Santos, A. Matijasevich, P. I. C. Lira e C. G. Victora recebem bolsa do Conselho Nacional de Desenvolvimento Científico e Tecnológico. C. C. Bortolotto recebeu bolsa da Coordenação de Aperfeiçoamento de Pessoal de Nível Superior (código 001).

\section{Referências}

1. Black MM, Walker SP, Fernald LCH, Andersen CT, DiGirolamo AM, Lu C, et al. Early childhood development coming of age: science through the life course. Lancet 2017; 389:77-90.

2. Fernandes M, Villar J, Stein A, Staines Urias E, Garza C, Victora CG, et al. INTERGROWTH21 st Project international INTER-NDA standards for child development at 2 years of age: an international prospective population-based study. BMJ Open 2020; 10:e035258.

3. Villar J, Fernandes M, Purwar M, StainesUrias E, Di Nicola P, Cheikh Ismail L, et al. Neurodevelopmental milestones and associated behaviours are similar among healthy children across diverse geographical locations. Nat Commun 2019; 10:511.

4. Rodriguez-Sibaja MJ, Villar J, Ohuma EO, Napolitano R, Heyl S, Carvalho M, et al. Fetal cerebellar growth and Sylvian fissure maturation: international standards from the Fetal Growth Longitudinal Study of the INTERGROWTH21(st) Project. Ultrasound Obstet Gynecol 2021; 57:614-23.

5. Wachs TD, Georgieff M, Cusick S, McEwen BS. Issues in the timing of integrated early interventions: contributions from nutrition, neuroscience, and psychological research. Ann N Y Acad Sci 2014; 1308:89-106.

6. Black MM, Behrman JR, Daelmans B, Prado EL, Richter L, Tomlinson M, et al. The principles of nurturing care promote human capital and mitigate adversities from preconception through adolescence. BMJ Global Health 2021; 6:e004436.

7. De-Regil LM, Peña-Rosas JP, Fernández-Gaxiola AC, Rayco-Solon P. Effects and safety of periconceptional oral folate supplementation for preventing birth defects. Cochrane Database Syst Rev 2015; (12):CD007950.

8. Hoddinott J, Maluccio JA, Behrman JR, Flores $R$, Martorell R. Effect of a nutrition intervention during early childhood on economic productivity in Guatemalan adults. Lancet 2008; 371:411-6.

9. Victora CG, Adair L, Fall C, Hallal PC, Martorell R, Richter L, et al. Maternal and child undernutrition: consequences for adult health and human capital. Lancet 2008; 371:340-57.

10. Trude ACB, Richter LM, Behrman JR, Stein AD, Menezes AMB, Black MM. Effects of responsive caregiving and learning opportunities during pre-school ages on the association of early adversities and adolescent human capital: an analysis of birth cohorts in two middle-income countries. Lancet Child Adolesc Health 2021; 5:37-46.

11. Sania A, Sudfeld CR, Danaei G, Fink G, McCoy DC, Zhu Z, et al. Early life risk factors of motor, cognitive and language development: a pooled analysis of studies from low/middleincome countries. BMJ Open 2019; 9:e026449. 
12. Sudfeld CR, McCoy DC, Danaei G, Fink G, Ezzati M, Andrews KG, et al. Linear growth and child development in low- and middle-income countries: a meta-analysis. Pediatrics 2015; 135:e1266-75.

13. Santos I, Munhoz T, Barcelos R, Blumenberg C, Bortolotto C, Matijasevich A, et al. Estudo de Linha de Base da Avaliação de Impacto do Programa Criança Feliz. Brasília: Secretaria de Avaliação e Gestão da Informação, Ministério da Cidadania; 2020. (Caderno de Estudos, 35).

14. Barros RP, Carvalho M, Mendonça R. Sobre as utilidades do Cadastro Único. Rio de Janeiro: Instituto de Pesquisa Econômica Aplicada; 2009.

15. Filgueiras A, Pires P, Maissonette S, Landeira-Fernandez J. Psychometric properties of the Brazilian-adapted version of the Ages and Stages Questionnaire in public child daycare centers. Early Hum Dev 2013; 89:561-76.

16. Fioravanti-Bastos ACM, Filgueiras A, Moura MLS. Evaluation of the Ages and Stages Questionnaire-Brazil by early childhood professionals. Estud Psicol (Campinas) 2016; 33:293-301.

17. Santana CMT, Filgueiras A, Landeira-Fernandez J. Ages \& Stages Questionnaire-Brazil-2011: adjustments on an early childhood development screening measure. Glob Pediatr Health 2015; 2:2333794X15610038.

18. Santos IS, Matijasevich A, Tavares BF, Barros AJD, Botelho IP, Lapolli C, et al. Validation of the Edinburgh Postnatal Depression Scale (EPDS) in a sample of mothers from the 2004 Pelotas Birth Cohort Study. Cad Saúde Pública 2007; 23:2577-88.

19. Matijasevich A, Munhoz TN, Tavares BF, Barbosa AP, Silva DM, Abitante MS, et al. Validation of the Edinburgh Postnatal Depression Scale (EPDS) for screening of major depressive episode among adults from the general population. BMC Psychiatry 2014; 14:284.

20. Villar J, Cheikh Ismail L, Victora CG, Ohuma EO, Bertino E, Altman DG, et al. International standards for newborn weight, length, and head circumference by gestational age and sex: the Newborn Cross-Sectional Study of the INTERGROWTH-21st Project. Lancet 2014; 384:857-68.

21. Villar J, Giuliani F, Fenton TR, Ohuma EO, Ismail LC, Kennedy SH. INTERGROWTH-21st very preterm size at birth reference charts. Lancet 2016; 387:844-5.

22. Harris PA, Taylor R, Thielke R, Payne J, Gonzalez N, Conde JG. Research electronic data capture (REDCap) - a metadata-driven methodology and workflow process for providing translational research informatics support. J Biomed Inform 2009; 42:377-81.

23. Shrier I, Platt RW. Reducing bias through directed acyclic graphs. BMC Med Res Methodol 2008; 8:70.
24. Victora CG, Huttly SR, Fuchs SC, Olinto MT. The role of conceptual frameworks in epidemiological analysis: a hierarchical approach. Int J Epidemiol 1997; 26:224-7.

25. Nilsen FM, Ruiz JDC, Tulve NS. A meta-analysis of stressors from the total environment associated with children's general cognitive ability. Int J Environ Res Public Health 2020; 17:5451.

26. Mensch BS, Chuang EK, Melnikas AJ, Psaki SR. Evidence for causal links between education and maternal and child health: systematic review. Trop Med Int Health 2019; 24:504-22.

27. Balaj M, York HW, Sripada K, Besnier E, Vonen HD, Aravkin A, et al. Parental education and inequalities in child mortality: a global systematic review and meta-analysis. Lancet 2021; 398:608-20.

28. Lohela TJ, Nesbitt RC, Pekkanen J, Gabrysch $\mathrm{S}$. Comparing socioeconomic inequalities between early neonatal mortality and facility delivery: cross-sectional data from 72 lowand middle-income countries. Sci Rep 2019; 9:9786.

29. Gakidou E, Cowling K, Lozano R, Murray CJ. Increased educational attainment and its effect on child mortality in 175 countries between 1970 and 2009: a systematic analysis. Lancet 2010; 376:959-74.

30. Victora CG, Horta BL, Mola CL, Quevedo L, Pinheiro RT, Gigante DP, et al. Association between breastfeeding and intelligence, educational attainment, and income at 30 years of age: a prospective birth cohort study from Brazil. Lancet Glob Health 2015; 3:e199-205

31. Stopa SR, Malta DC, Monteiro CN, Szwarcwald CL, Goldbaum M, Cesar CLG. Use of and access to health services in Brazil, $2013 \mathrm{Na}$ tional Health Survey. Rev Saúde Pública 2017; 51 Suppl 1:3s.

32. Disease GBD, Injury I, Prevalence C. Global, regional, and national incidence, prevalence, and years lived with disability for 354 diseases and injuries for 195 countries and territories, 1990-2017: a systematic analysis for the Global Burden of Disease Study 2017. Lancet 2018; 392:1789-858.

33. Salk RH, Hyde JS, Abramson LY. Gender differences in depression in representative national samples: meta-analyses of diagnoses and symptoms. Psychol Bull 2017; 143:783-822.

34. Letourneau NL, Dennis CL, Cosic N, Linder $\mathrm{J}$. The effect of perinatal depression treatment for mothers on parenting and child development: a systematic review. Depress Anxiety 2017; 34:928-66.

35. Slomian J, Honvo G, Emonts P, Reginster JY, Bruyere O. Consequences of maternal postpartum depression: a systematic review of maternal and infant outcomes. Womens Health (Lond) 2019; 15:1745506519844044. 
36. Woldeyohannes D, Tekalegn Y, Sahiledengle B, Ermias D. The effect of postpartum depression on infant feeding practice in Sub-Saharan African Countries: systematic review and metaanalysis. BMC Pregnancy Childbirth 2021; 21:113.

37. Dadi AF, Miller ER, Mwanri L. Postnatal depression and its association with adverse infant health outcomes in low- and middle-income countries: a systematic review and meta-analysis. BMC Pregnancy Childbirth 2020; 20:416.

38. Goodman SH, Simon HF, Shamblaw AL, Kim CY. Parenting as a mediator of associations between depression in mothers and children's functioning: a systematic review and metaanalysis. Clin Child Fam Psychol Rev 2020; 23:427-60.

39. Liu Y, Kaaya S, Chai J, McCoy DC, Surkan PJ, Black MM, et al. Maternal depressive symptoms and early childhood cognitive development: a meta-analysis. Psychol Med 2017; 47:680-9.

40. Ferrari AJ, Somerville AJ, Baxter AJ, Norman $\mathrm{R}$, Patten SB, Vos T, et al. Global variation in the prevalence and incidence of major depressive disorder: a systematic review of the epidemiological literature. Psychol Med 2012; 43:471-81.

41. Slomian J, Honvo G, Emonts P, Reginster J-Y, Bruyère $\mathrm{O}$. Consequences of maternal postpartum depression: a systematic review of maternal and infant outcomes. Womens Health (Lond) 2019; 15:1745506519844044.

42. Lovejoy MC, Graczyk PA, O'Hare E, Neuman G. Maternal depression and parenting behavior: a meta-analytic review. Clin Psychol Rev 2000; 20:561-92.
43. Dawson G, Frey K, Panagiotides H, Osterling J, Hessl D. Infants of depressed mothers exhibit atypical frontal brain activity: a replication and extension of previous findings. J Child Psychol Psychiatry 1997; 38:179-86.

44. Wen DJ, Soe NN, Sim LW, Sanmugam S, Kwek $\mathrm{K}$, Chong YS, et al. Infant frontal EEG asymmetry in relation with postnatal maternal depression and parenting behavior. Transl Psychiatry 2017; 7:e1057.

45. Tierney AL, Nelson 3rd CA. Brain development and the role of experience in the early years. Zero Three 2009; 30:9-13.

46. Boggs D, Milner KM, Chandna J, Black M, Cavallera V, Dua T, et al. Rating early child development outcome measurement tools for routine health programme use. Arch Dis Child 2019; 104 Suppl 1:S22-33.

47. Silva MA, Mendonça Filho EJ, Mônego BG, Bandeira DR. Instruments for multidimensional assessment of child development: a systematic review. Early Child Dev Care 2018; 190:1257-71.

48. Brasil. Lei 13.257, de 8 de março de 2016. Dispõe sobre as políticas públicas para a primeira infância e altera a Lei no 8.069, de 13 de julho de 1990 (Estatuto da Criança e do Adolescente), o Decreto-Lei no 3.689, de 3 de outubro de 1941 (Código de Processo Penal), a Consolidação das Leis do Trabalho (CLT), aprovada pelo Decreto-Lei no 5.452, de 1o de maio de 1943 , a Lei no 11.770 , de 9 de setembro de 2008, e a Lei no 12.662 , de 5 de junho de 2012. Diário Oficial da União 2016; 9 mar. 
Abstract

The study aimed to assess socioeconomic, family, and individual factors associated with infant development (i.e., in the first year of life) among families with social vulnerability. This was a cross-sectional analysis of baseline data from a randomized trial. The study included 3,242 children $<12$ months of age living in 30 municipalities from five regions of Brazil. The choice of states and municipalities was intentional, based on the implementation of the Brazilian Happy Child Program. The sample was selected among eligible children for the Brazilian Happy Child Program, and the objective was the promotion of infant development. The Ages and Stages Questionnaire (ASQ) was used to assess infant development. A three-level analytical model (state, municipality, and individuals), using the Wald test for heterogeneity and linear trend, estimated the mean ASQ-3 and 95\% confidence interval (95\%CI). The analyses were adjusted for potential confounders. Information was analyzed for 3,061 (94.4\%) children with available data for ASQ-3. Infant development scores (total and in all the domains) were some $12 \%$ lower in preterm children and those with intrauterine growth restriction (small for gestational age). Lower scores were seen in children of mothers with low schooling, depressive symptoms, two or more children under seven years of age living in the household, and who did not report selfperceived support or help during the pregnancy. In conclusion, potentially modifiable characteristics (schooling, maternal depression, and prematurity/ intrauterine growth restriction) showed greater impact on reducing the infant development score in all the target domains.

Child Development; Social Determinants of Health; Development Disabilities; Depression; Pervasive Child Development Disorders

\section{Resumen}

El objetivo fue evaluar los factores socioeconómicos, familiares e individuales, asociados al desarrollo infantil en el primer año de vida, entre familias con vulnerabilidad social. Se trata de un análisis transversal, con datos de la base de referencia de un ensayo aleatorio. El estudio incluyó a 3.242 niños < 12 meses de edad, residentes en 30 municipios de cinco regiones de Brasil. La elección de estados y municipios fue intencional, considerando como base la implementación del Programa Niño Feliz. La muestra se seleccionó a partir de niños elegibles para el Programa Niño Feliz, cuyo objetivo es promover la estimulación y el desarrollo infantil. Se utilizó el Ages and Stages Questionnaire (ASQ) para la evaluación del desarrollo infantil. Un modelo de análisis multinivel en tres niveles (estado, municipio e individuos), usando el test de Wald para la heterogeneidad y tendencia lineal, estimó la media del ASQ-3 y el intervalo de 95\% de confianza (IC95\%). Los análisis se ajustaron para potenciales factores de confusión. Se analizó información de 3.061 (94,4\%) niños con datos disponibles para ASQ-3. Las puntuaciones de desarrollo infantil (total y en todos los dominios) fueron cerca de un 12\% menores en niños nacidos pretérmino y con restricción del crecimiento intrauterino (pequeños para la edad gestacional). Se observaron menores puntuaciones en hijos de madres con baja escolaridad, con síntomas de depresión, con dos o más niños menores de siete años residiendo en el domicilio y que no informaron autopercepción de apoyo/ayuda durante la gestación. Se concluye que las características potencialmente modificables (escolaridad, depresión materna y prematuridad/restricción del crecimiento intrauterino) presentaron un mayor impacto en la reducción de la puntuación de desarrollo en todos los dominios evaluados.

Desarrollo Infantil; Determinantes Sociales de la Salud; Discapacidades del Desarrollo; Depresión; Trastornos Generalizados del Desarrollo Infantil
Recebido em 05/Nov/2020

Versão final reapresentada em 23/Jul/2021

Aprovado em 24/Jul/2021 